\title{
3D Open-Surface Shape Correspondence for Statistical Shape Modeling: Identifying Topologically Consistent Landmarks
}

\author{
Pahal Dalal ${ }^{1}$, Lili Ju${ }^{2}$, Michael McLaughlin ${ }^{1}$, Xiangrong $Z_{\text {hou }}^{3}$, Hiroshi Fujita ${ }^{3}$, and Song Wang ${ }^{1, *}$ \\ ${ }^{1}$ Department of Computer Science \& Engineering, University of South Carolina, Columbia, SC, USA \\ ${ }^{2}$ Department of Mathematics, University of South Carolina, Columbia, SC, USA \\ ${ }^{3}$ Department of Intelligent Image Information, Gifu University, Japan
}

\begin{abstract}
Shape correspondence, which aims at accurately identifying corresponding landmarks from a given population of shape instances, is a very challenging step in constructing a statistical shape model such as the Point Distribution Model. The state-of-the-art methods such as MDL and SPHARM are primarily focused on closed-surface shape correspondence. In this paper we develop a novel method aimed at identifying accurately corresponding landmarks on 3D open-surfaces with a closed boundary. In particular, we enforce explicit topology consistency on the identified landmarks to ensure that they form a simple, consistent triangle mesh to more accurately model the correspondence of the underlying continuous shape instances. The proposed method also ensures the correspondence of the boundary of the open surfaces. For our experiments, we test the proposed method by constructing a statistical shape model of the human diaphragm from 26 shape instances.
\end{abstract}

\section{Introduction}

The Point Distribution Model (PDM) [9] has become a very popular tool for statistical shape analysis and has been widely used in various computer-vision and medicalimaging applications such as image segmentation $[9,23]$ and shape based diagnosis $[3,4,8,15,21]$. The major challenge in constructing a PDM comes from the step of (landmark-based) shape correspondence, which aims at identifying a set of accurately corresponding landmarks from a population of given shape instances. The nonlinearity of the shape description and shape variation for most anatomical structures leads to a problem where it is very difficult to find an optimal solution. This difficulty is further increased when the shape is a 3D surface.

${ }^{*}$ Corresponding author. Email: songwang@cec.sc.edu. This work was funded, in part, by AFOSR FA9550-07-1- 0250.
In the past several years, many 3D shape correspondence methods have been proposed for PDM construction $[6,7,11,19,22,26,28,34]$. Most of these methods are aimed at closed-surface shape correspondence. For example, both the Minimum Description Length (MDL) [11, 18] and Spherical Harmonics (SPHARM) [5, 16] methods map each shape instance to a sphere and reduce the shape correspondence problem to that of parameterizing the sphere. It is usually difficult to apply such a sphere-mapping step to open-surface shapes.

In many medical-imaging applications, the structure of interest bears an open-surface shape with a defined, closed boundary. The human diaphragm is a typical example of such shapes. It is an open-surface tissue that separates the chest and abdomen. It is located beneath the lung and above the liver, with its edge affixed to the corresponding bone frame (ribs, xiphoid and lumbar), forming a 3D closed boundary, which corresponds across all shape instances. This is different from the open surface resulting from the cropping of a 3D object by image perimeter, where the surface boundary is usually of a 2D form and may not correspond across all shape instances [27]. The shape and position of the diaphragm have been related to pulmonary function in clinical applications. However, the analysis of the diaphragm is currently hindered by the nature of the underlying shape which is unsuitable for existing shape correspondence methods. Hence, we need a method to perform the shape correspondence of an open-surface shape with a closed boundary to construct a PDM.

Related to this paper is the 3D landmark-sliding method SLIDE [10] that can be used to correspond both opensurface and closed-surface shape instances. In this method, each target shape instance is first aligned with a given template shape. Based on this alignment, an initial estimate of the corresponding landmarks is established and a landmarksliding framework is developed to refine the initial estimates of corresponding landmarks. Compared to MDL and SPHARM, this method requires less CPU time and usually leads to a PDM with better compactness, generality and 
specificity characteristics [10]. However, this method does not guarantee that the landmarks identified across shape instances have consistent topology. Further, it does not ensure that landmarks along the closed boundary of the template shape correspond to landmark points along the closed boundary of the target shape. Also related to this paper are the works on brain mapping $[13,14,30]$, which address the problem of identifying corresponded landmarks on cortical surfaces, which are open surfaces. However, these works are highly dependent on the geometric information of anatomic features such as the gyra and sulci. In this paper, we address the problem of identifying corresponding landmarks on smooth surfaces which may have few (or none) such anatomic features with defined geometric information.

In this paper, we develop a new method to correspond 3D open-surface shape instances by identifying topologically consistent landmarks. Specifically, we define landmarktopology consistency through the triangle mesh representation of a surface: the landmarks identified from each shape instance must constitute a valid triangle mesh for this shape instance when these landmarks are connected in the same order as in a given template. Starting with a set of 3D open-surface shape instances in the form of densely sampled point clouds, the proposed method consists of the following steps: (a) Construct a 3D triangle mesh for each shape instance independently. (b) Map each constructed triangle mesh to a $2 \mathrm{D}$ planar triangle mesh using conformal mapping [1]. (c) Construct an initial estimate of topologically consistent landmarks in the 2D plane followed by inverse conformal mapping to the 3D shape instance. (d) Apply a landmark-sliding algorithm to refine the landmarks while maintaining the topology consistency.

The remainder of the paper is organized as follows: In Section 2, we formulate the problem by describing the concepts of topology consistency and shape correspondence error. Section 3 describes in detail the proposed method. We present our experiments and results in Section 4, followed by a brief conclusion.

\section{Problem Description}

The input of shape correspondence is a set of shape instances. As shown in Fig. 1, we represent each open-surface shape instance $S$ that describes an open-surface shape as: (a) $S_{P}$, a dense point cloud defining the entire open surface; (b) $S_{B}$, the subset of $S_{P}$ that describe the closed boundary of the surface; (c) $S_{M}$, a triangle mesh constructed to approximate $S$; (d) $S_{L}$, the set of landmarks identified by the shape correspondence method; (e) $S_{T}$, the triangle mesh generated by using $S_{L}$. Different from $S_{L}$ and $S_{T}, S_{M}$ is constructed independently and may contain different number of vertices and triangles for each shape instance.

Among these, $S_{P}$ is the original data obtained from manually or automatically segmented medical images. $S_{B}$ and
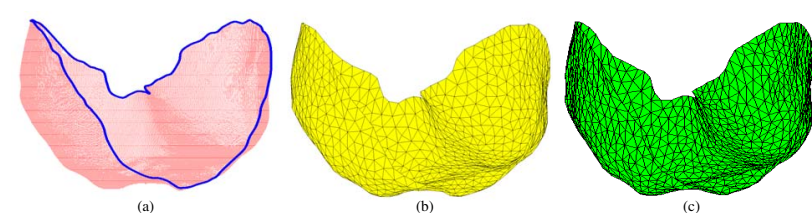

Figure 1. Illustration of each representation of a shape: (a) The red points are the point cloud $S_{P}$ and the blue points are the boundary points $S_{B}$. (b) The discrete triangle mesh $S_{M}$ to approximate the surface $S$. (c) The landmark triangle mesh $S_{T}$. Each vertex is a landmark in $S_{L}$. Note that $S_{M}$ and $S_{T}$ are not the same.

$S_{M}$ are derived from $S_{P}$ to describe the boundary feature of the surface and topological nature of the open-surface shape instance. We use $S_{M}$ to construct the conformal 2D planar mapping of the surface $S . S_{L}$ and $S_{T}$ are the output of the proposed method. As in previous research on PDM-based shape correspondence [3, 4, 9], the identified landmarks $S_{L}$ may not coincide with any anatomically significant features.

We simplify the problem formulation to correspond two shape instances: a template $U$ and a target $V$. For multiple shape correspondence, we can select one shape as the template and repeatedly correspond each of the remaining ones to this template. For the template $U$, we can construct the triangle mesh $U_{M}$ independent of all other shape instances. We directly set the vertices of $U_{M}$ to be the landmarks $U_{L}$, which are fixed in shape correspondence and set the triangle mesh $U_{T}=U_{M}$, which defines the triangulation information of connecting identified landmarks into a triangle mesh in the target shape instance. The problem can then be formulated as constructing a corresponding set of landmarks $V_{L}$ on the target surface $V$ that minimize a pre-defined shape-correspondence error $d\left(U_{L}, V_{L}\right)$. In addition, from $V_{L}$ we can construct a mesh $V_{T}$ by applying the same vertex triangulation information as in $U_{T}$. We say that $V_{L}$ is topologically consistent with $U_{L}$, if $V_{T}$ is a valid and accurate triangle mesh representation of $V$. Particularly, $V_{T}$ must be a simple mesh without any self intersections. In this paper, we denote each landmark in $U_{L}$ to be $\mathbf{u}_{i}=\left(u_{i x}, u_{i y}, u_{i z}\right)$ and the corresponding landmark in the target $V_{L}$ to be $\mathbf{v}_{i}=\left(v_{i x}, v_{i y}, v_{i z}\right), i=1,2, \ldots, n$.

\subsection{Shape Correspondence Error}

We use 3D thin-plate splines $[3,12,31]$ to model the correspondence error between template landmarks $U_{L}$ and target landmarks $V_{L}$. The thin-plate spline bending energy measures the energy required to deform a volume to match these two sets of landmarks. In particular, the thin-plate spline transform finds a mapping $\mathbf{t}=\left(t_{x}, t_{y}, t_{z}\right)$ from $U_{L}$ to $V_{L}$, i.e. $v_{i x}=t_{x}\left(\mathbf{u}_{i}\right), v_{i y}=t_{y}\left(\mathbf{u}_{i}\right)$ and $v_{i z}=t_{z}\left(\mathbf{u}_{i}\right)$ for $i=1,2, \ldots, n$. The thin-plate bending energy is invariant under any affine transformation. This is suitable for our purpose because we wish to model the non-rigid deformation observed in biological shapes. 
Further, we find an expectation, $V_{E}$, for the target landmarks. Let the expected target landmarks be denoted by $\mathbf{v}_{i}^{e}=\left(v_{i x}^{e}, v_{i y}^{e}, v_{i z}^{e}\right), i=1,2, \ldots, n$. In this paper we obtain $V_{E}$ by using a simple feature of the boundary of opensurface shapes, i.e. the boundary of open-surface shapes must be corresponded. The surface boundaries of the template and target can be derived from point sets $U_{B}$ and $V_{B}$, respectively. Both of them are a $3 \mathrm{D}$ closed contour. Note that the landmarks on the template boundary are fixed. They are the $m$ surface boundary points in $U_{L}$ and our goal is to identify $m$ corresponded landmarks along the surface boundary of $V$. For simplicity, we denote the $m$ surface boundary points on the template to be $U_{B L}$ and the identified $m$ corresponded landmarks on the target to be $V_{B L}$. In this paper, we directly extend the 2D landmark-sliding algorithm [33] to correspond these two 3D curves. The only extension is to use 3D thin-plate bending energy for describing the shape-correspondence error. We derive the thin-plate transform $\mathbf{t}$ from the corresponded boundary landmarks and then apply it to all the landmarks in $U_{L}$ to construct $V_{E}=$ $\mathbf{t}\left(U_{L}\right)$.

Hence, the shape correspondence error may be defined in a quadratic form as:

$$
\begin{aligned}
& d\left(U_{L}, V_{L}\right)= \\
& \mathbf{v}_{x}^{T} \mathbf{L} \mathbf{v}_{x}+\mathbf{v}_{y}^{T} \mathbf{L} \mathbf{v}_{y}+\mathbf{v}_{z}^{T} \mathbf{L} \mathbf{v}_{z} \\
& +\sum_{i=1}^{n} \lambda_{i}\left\|\mathbf{v}_{i}-\mathbf{v}_{i}^{e}\right\|^{2}
\end{aligned}
$$

where $\mathbf{v}_{x}, \mathbf{v}_{y}$ and $\mathbf{v}_{z}$ are the columnized vectors that contain the $x, y$ and $z$ coordinates of the landmarks in $V_{L}$, respectively. $\mathbf{L}$ is the $n \times n$ upper left submatrix of

$$
\left[\begin{array}{ll}
\mathbf{K} & \mathbf{D} \\
\mathbf{D}^{T} & 0
\end{array}\right]^{-1}
$$

where the $n \times n$ matrix $\mathbf{K}$ has elements $k_{i j}=-\frac{1}{8 \pi} \| \mathbf{u}_{i}-$ $\mathbf{u}_{j} \|$ and the $n \times 4$ matrix $\mathbf{D}=\left[\mathbf{1}_{n \times 1}, \mathbf{u}_{x}, \mathbf{u}_{y}, \mathbf{u}_{z}\right]$ with $\mathbf{u}_{x}, \mathbf{u}_{y}$ and $\mathbf{u}_{z}$ being the columnized vectors that contain the $x, y$ and $z$ coordinates of the landmarks in $U_{L}$. Since $V_{E}$ is obtained by simply using the correspondence of the boundary, we set $\lambda_{i}>0$ for all $\mathbf{v}_{i} \in V_{B L}$, and $\lambda_{i}=0$ otherwise. In this way, the right side of (1) includes the $3 \mathrm{D}$ thin-plate spline bending energy in the first three terms and the last term penalizes the error between the boundary landmarks of the template and target shapes.

\subsection{Topology Consistency of Landmarks}

Using the thin-plate bending energy as the shape correspondence error, does not guarantee the preservation of topology consistency between the template and the target [32]. For example, in Fig. 2, the landmarks $V_{L}$ shown in (b) have a finite bending energy with the template landmarks $U_{L}$ in (a). However, when we connect the landmarks $V_{L}$ by following the same vertex connectivity as the template triangle mesh, we obtain a non-simple triangle mesh $V_{T}$ with self intersections. Note that we cannot redefine the connection order in $V_{T}$ to remove self intersections because this connection order is defined by the template mesh $U_{T}$.

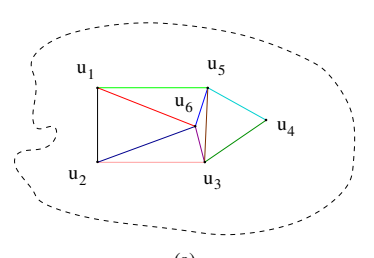

(a)

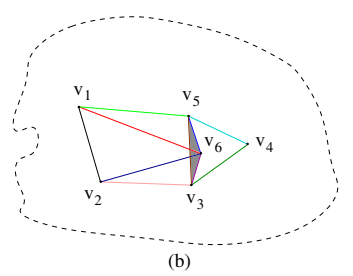

(b)
Figure 2. Illustration of inconsistency in topology of template landmarks $U_{L}$ and target landmarks $V_{L}$ : (a) Triangulation of template landmarks $\mathbf{u}_{1}, \mathbf{u}_{2}, \mathbf{u}_{3}, \mathbf{u}_{4}, \mathbf{u}_{5}, \mathbf{u}_{6}$. (b) Triangulation of corresponding target landmarks $\mathbf{v}_{1}, \mathbf{v}_{2}, \mathbf{v}_{3}, \mathbf{v}_{4}, \mathbf{v}_{5}, \mathbf{v}_{6}$ showing self-intersection.

Landmark topology consistency reflects the geometric homeomorphism between the template and the target shape instances. Hence, landmark topology consistency should be included as a critical constraint in shape correspondence and statistical shape modeling. In Section 3, we introduce a new approach based on conformal mapping to explicitly preserve the landmark topology consistency in open-surface shape correspondence.

\section{Proposed Method}

The proposed method consists of three main steps: First, we construct the template landmarks $U_{L}$ and the template triangle mesh $U_{T}$ from the dense point cloud $U_{P}$. Particularly, we adapt the K-means clustering algorithm [17] to preserve the surface boundary in $U_{T}$. The same clustering method is independently applied on the point cloud $V_{P}$ to construct a triangle mesh $V_{M}$ for the target $V$. Second, we derive an initial estimate of corresponded target landmarks $V_{L}$ by applying the shape-context [2] method to $\tilde{U}_{T}$ and $\tilde{V}_{M}$, the 2D conformal mappings of $U_{T}$ and $V_{M}$, respectively. Finally, we develop a new aggressive landmark sliding algorithm to refine $V_{L}$ by minimizing the shape correspondence error $d\left(U_{L}, V_{L}\right)$.

\subsection{Construction of $U_{L}, U_{T}$ and $V_{M}$}

Many methods have been developed for constructing a triangle mesh from a dense point cloud. In this paper, we use an adaptation of the well known K-means clustering algorithm [17] on the dense point cloud. After this, we treat the sparsely and uniformly (or nearly uniformly) distributed cluster centers as the vertices to construct a triangle mesh.

The traditional K-means clustering algorithm cannot accurately preserve the boundary of an open-surface: In the 


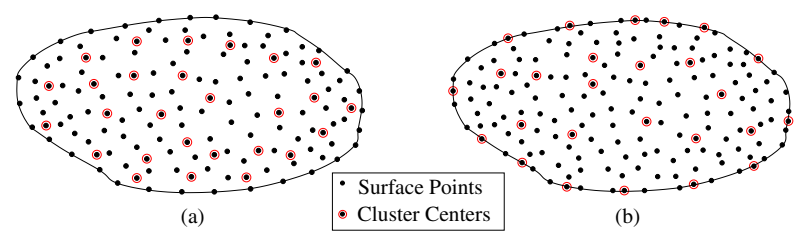

Figure 3. A simple 2D illustration of the constrained K-means clustering algorithm: (a) Traditional K-means clustering of $S_{P}$ leads to well distributed cluster centers, but surface boundary points are not usually included as cluster centers. (b) Constrained $\mathrm{K}$-means clustering with some cluster centers along the boundary.

K-means algorithm, the cluster centers are derived by averaging the points in a neighborhood and there is no guarantee that sufficient points along the surface boundary can be included as cluster centers. An example is shown in Fig. 3 (a), where no points along the surface boundary are included as cluster centers (circled dots). To address this problem, we adapt the K-means algorithm to a constrained K-means algorithm, which identifies sufficient cluster centers from the surface boundary as shown in Fig. 3 (b).

As in the traditional K-means clustering algorithm, we alternately update the cluster centers and the point assignments in each cluster, but add one more step. For the template $U$, in each iteration of updating the cluster centers, we first estimate the $K$ cluster centers by averaging the points in each cluster. After this, we further update the cluster centers that contain points in $U_{B}$ (the surface-boundary points). Specifically, if a cluster contains any point in $U_{B}$, we update the center of this cluster by averaging only the surfaceboundary points in this cluster. We alternately repeat this two-step cluster-center updating operation and the pointassignment operation until there is no significant change in the positions of the cluster centers. Using this constrained $\mathrm{K}$-means clustering algorithm, we can construct a triangle mesh $U_{T}$ that well represents the underlying surface.

Using the same method, we also independently process the point cloud of each target shape $V$ to construct a triangle mesh $V_{M}$. Note that, there is no correspondence between the vertices of $U_{T}$ and $V_{M}$ because we process them independently. In fact, $U_{T}$ and $V_{M}$ may have different number of vertices. In the next section, we develop a method to derive landmarks $V_{L}$ and mesh $V_{T}$ from the target so that they correspond to the landmarks $U_{L}$ and mesh $U_{T}$ on the template.

\subsection{Initial Estimate of $V_{L}$}

We use shape matching based on geometric information along with conformal mapping to construct an initial estimate for the target landmarks $V_{L}$. First, we use the Least Squares Conformal Mapping (LSCM) method of [24] to flatten the template triangle mesh $U_{T}$ and target triangle mesh $V_{M}$ to their 2D conformal representations $\tilde{U}_{T}$ and $\tilde{V}_{M}$ respectively, as shown in Fig. 4 (a) and (b). With the 2D conformal maps, we can easily build a 2D parametrization for the 3D surface.

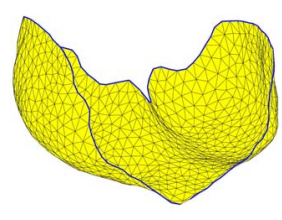

(a)

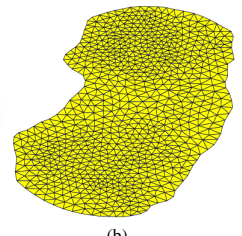

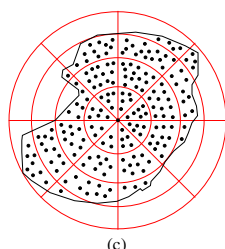

Figure 4. An illustration of LSCM conformal mapping and shapecontext descriptor: (a) A 3D mesh of the human diaphragm. (b) The LSCM conformal map of the 3D mesh. (c) The shape-context of a landmark $\mathbf{u}_{i}$

Second, we use the correspondence between the boundary landmarks $\tilde{U}_{B L}$ and $\tilde{V}_{B L}$ to eliminate the translation, rotation and scaling differences between the template and the target using Procrustes analysis on the 2D plane. We then use the shape-context method [2] to find a matching between all the template landmarks $\tilde{U}_{L}$ (on the 2 D plane) and $\tilde{V}_{D}$, a down-sampled subset of points on the target on the 2D plane. We use the same method described in Section 3.1 to preserve the boundary while constructing $\tilde{V}_{D}$. We only require that the number of points in $\tilde{V}_{D}$ be larger than or equal to the number of landmarks in $\tilde{U}_{L}$ so that each template landmark is assigned a corresponding target landmark. To enforce the requirement that template boundary landmarks $U_{B L}$ are corresponded to a point along $V_{B}$, we set the cost of matching boundary landmarks with non-boundary points to a very large number. After finding the matches of $\tilde{U}_{L}$ in $\tilde{V}_{D}$, we perform inverse conformal mapping to get a set of target landmarks on the target.

However, we cannot directly use this set of landmarks on the target as the initial estimate of $V_{L}$. It may contain mismatches because shape context only considers geometry information but not spatial topology, as shown in Fig. 4 (c). If we construct the triangle mesh $V_{T}$ by connecting $V_{L}$ using the same connection information as $U_{T}$, the mesh $V_{T}$ may not be a simple mesh without self-intersections. We use statistical regression and develop a process of elimination to remove such pairs $\left(\mathbf{u}_{i}, \mathbf{v}_{i}\right)$. We then use the remaining matched pairs to establish a topologically consistent initial estimate for $V_{L}$.

Specifically, we define the regression cost function as

$$
\phi=\sum_{i=1}^{n}\left\|\mathbf{v}_{i}-\hat{\mathbf{v}}_{i}\right\|^{2}+\mathbf{v}_{x}^{T} \mathbf{L} \mathbf{v}_{x}+\mathbf{v}_{y}^{T} \mathbf{L} \mathbf{v}_{y}+\mathbf{v}_{z}^{T} \mathbf{L} \mathbf{v}_{z}
$$

where $\mathbf{L}$ is the same thin-plate spline bending matrix as defined in (1). We calculate the optimal values of $\hat{\mathbf{v}}_{i}$ to minimize this cost by equating to 0 , the partial derivative of (2) with respect to $\hat{\mathbf{v}}$. To identify the mismatched pairs $\left(\mathbf{u}_{i}\right.$, 
$\mathbf{v}_{i}$ ), we calculate the Euclidean distance $r_{i}$ between each $\mathbf{v}_{i}$ and $\hat{\mathbf{v}}_{i}$. Finally we sort the list of pairs $\left(\mathbf{u}_{i}, \mathbf{v}_{i}\right)$ by decreasing value of $r_{i}$ associated with $\mathbf{v}_{i}$ (worst matched pair to best). We discard the pair $\left(\mathbf{u}_{i}, \mathbf{v}_{i}\right)$ if it satisfies the following conditions: (i) The pair violates the topology consistency requirement. For example, in Fig. 2 we discard the pair $\left(\mathbf{u}_{6}, \mathbf{v}_{6}\right)$. (ii) They are not boundary landmarks. However, neighboring boundary landmarks may be switched to ensure topology consistency. (iii) $30 \%$ pairs with the largest value of $r_{i}$ are considered as mismatches, but we do not remove all the landmarks on the same triangle in $U_{T}$.

By this process of elimination, we get $U_{R}$ and $V_{R}$ which are topologically consistent and representative landmarks. Finally, we calculate the 3D thin-plate transform between $U_{R}$ and $V_{R}$, such that $V_{R}=\mathbf{t}\left(U_{R}\right)$ and apply this transform $\mathbf{t}$ to all landmarks in $U_{L}$ to get the initial estimate for $V_{L}$ i.e. $V_{L}=\mathbf{t}\left(U_{L}\right)$. We now connect the landmarks in $V_{L}$ using the same triangulation information as $U_{T}$ to get $V_{T}$.

\subsection{Refining $V_{L}$ by Aggressive Landmark Sliding}

The initial estimate $V_{L}$ described above is constructed in the $2 \mathrm{D}$ conformal map domain using a shape-matching based approach. While it preserves the landmark-topology consistency, it may not minimize the 3D shape correspondence error (1). In this section, we adapt the 3D landmark-sliding algorithm proposed in [10] to further refine $V_{L}$. In this algorithm, all landmarks in $V_{L}$ are simultaneously and iteratively moved on the surface $V$ to minimize the correspondence error (1). In every iteration, we first move each landmark on its tangent plane and then project the new landmarks back onto the surface $V$. More specifically, each landmark $\mathbf{v}_{i} \in V_{L}$ is moved to $\mathbf{v}_{i}^{\prime}=\mathbf{v}_{i}+\alpha_{i} \mathbf{p}_{i}+\beta_{i} \mathbf{q}_{i}$ on the tangent plane, where $\mathbf{p}_{i}$ and $\mathbf{q}_{i}$ are linearly independent unit tangent vectors and $\alpha_{i}$ and $\beta_{i}$ are the sliding distances along these two tangent vectors. The optimal sliding distances can be found by solving the following quadratic-programming problem

$$
\begin{aligned}
& \min _{\boldsymbol{\alpha}, \boldsymbol{\beta}} \phi\left(U_{L}, V_{L} ; \boldsymbol{\alpha}, \boldsymbol{\beta}\right)= \\
& \sum_{\star \in\{x, y, z\}}\left(\mathbf{v}_{\star}+\mathbf{P}_{\star} \boldsymbol{\alpha}+\mathbf{Q}_{\star} \boldsymbol{\beta}\right)^{T} \mathbf{L}\left(\mathbf{v}_{\star}+\mathbf{P}_{\star} \boldsymbol{\alpha}+\mathbf{Q}_{\star} \boldsymbol{\beta}\right) \\
& +\sum_{i=1}^{n} \lambda_{i}\left\|\mathbf{v}_{i}+\mathbf{p}_{i} \boldsymbol{\alpha}_{i}+\mathbf{q}_{i} \boldsymbol{\beta}_{i}-\mathbf{v}_{i}^{e}\right\|^{2}
\end{aligned}
$$

subject to constraint

$$
\left|\alpha_{i}\right| \leq \epsilon,\left|\beta_{i}\right| \leq \epsilon
$$

where,

$$
\begin{aligned}
& \boldsymbol{\alpha}=\left(\alpha_{1}, \ldots, \alpha_{n}\right)^{T}, \quad \boldsymbol{\beta}=\left(\beta_{1}, \beta_{2}, \ldots, \beta_{n}\right)^{T} \\
& \mathbf{P}_{\star}=\operatorname{diag}\left(\mathbf{p}_{1 \star}, \mathbf{p}_{2 \star}, \ldots, \mathbf{p}_{n \star}\right), \\
& \mathbf{Q}_{\star}=\operatorname{diag}\left(\mathbf{q}_{1 \star}, \mathbf{q}_{2 \star}, \ldots, \mathbf{q}_{n \star}\right) . \\
& \lambda_{i}>0, \text { if } \mathbf{v}_{i} \text { is a boundary landmark } \\
& \lambda_{i}=0, \text { otherwise. }
\end{aligned}
$$

Note that the second term of (3) only considers the expected boundary landmarks since $\lambda_{i}=0$ for all other landmarks. This construction ensures that the boundary landmarks do not move far from the surface boundary.

The constraint (4) is the same as the one used in [10] for controlling the step length in sliding. However, in this paper, we introduce aggressive landmark sliding. We first set $\epsilon$ to a large value and perform a step of landmark sliding. If the topology consistency is broken by the result of this step of sliding, we replace $\epsilon=\epsilon / 2$ and redo the step of landmark sliding. We continue in this fashion until a value for $\epsilon$ is reached such that the landmark sliding can be performed without violating the topology consistency or the value of $\epsilon$ becomes too small to perform any landmark sliding. This is in contrast to the method used in [10] where the landmark sliding is restricted to a very small region by setting $\epsilon$ to a fixed, small value. For projecting $\mathbf{v}_{i}^{\prime}$ back onto the surface $V$, we find, from the input dense point cloud $V_{P}$, a point that has the smallest distance to $\mathbf{v}_{i}^{\prime}$. The resulting closest point is then used as $\mathbf{v}_{i}$ for the next iteration of landmark sliding.

Generally, it is very difficult to directly check the topology consistency of landmarks in 3D. We utilize the 2D parametrization rendered by the conformal mapping. Specifically, we find, from triangle mesh $V_{M}$, the closest point to $\mathbf{v}_{i}^{\prime}$. This point denoted as $\mathbf{v}_{i}^{\prime \prime}$, may be located within a triangle or along the sides of a triangle. Since $\mathbf{v}_{i}^{\prime \prime}$, $i=1,2, \ldots, n$ are on the triangle mesh $V_{M}$, we can apply the conformal mapping to find their mapping $\tilde{\mathbf{v}}_{i}^{\prime \prime}$. If the triangle mesh built on the point-set $\tilde{\mathbf{v}}_{i}^{\prime \prime}, i=1,2, \ldots, n$ using the same connection order as in $U_{T}$ has any selfintersections, we know the landmark topology is inconsistent with the template mesh $U_{T}$.

\section{Experiments}

We perform our experiments on a set of 26 human diaphragm shape instances, each described by approximately 100,000 to 250,000 points. Identifying the diaphragm from torso CT images is a very important task in computer-aided diagnosis [35]. One of the reasons for the difficulty in performing shape correspondence on the diaphragm is the acquisition of input data. This is due to the nature of the region near the boundary of the diaphragm which is very cluttered in CT images and difficult to label. We obtained the 26 diaphragm shapes as follows: First CT images of the torso 
are obtained while the subject is holding their breath such that the lung volume is as large as possible for easy detection. Next, a semi-automatic method is used to roughly segment the diaphragm shape from the CT images. Finally, the shape is manually updated by adding, deleting and moving the identified points. This is a very tedious and time consuming task. In the future, we plan to use the proposed method to construct and apply a PDM to facilitate the automatic segmentation of the diaphragm.

In our experiments, we use the proposed method and SLIDE to correspond the same 26 diaphragm shape instances and perform a comparison. We then construct and evaluate the point distribution model (PDM) defined by the mean shape $\mu$ and covariance matrix derived from the correspondence results. For the proposed method, we set the initial value of $\epsilon=50$ in (4). Landmark sliding is set to stop after 20 iterations and we identify 842 corresponded landmarks on each of the 26 diaphragm shape instances. SLIDE was used with the recommended default parameters and asked to identify the same landmarks by selecting the same template shape for both methods. This ensures that the mesh connecting landmarks in the proposed method can be used to connect landmarks for SLIDE.

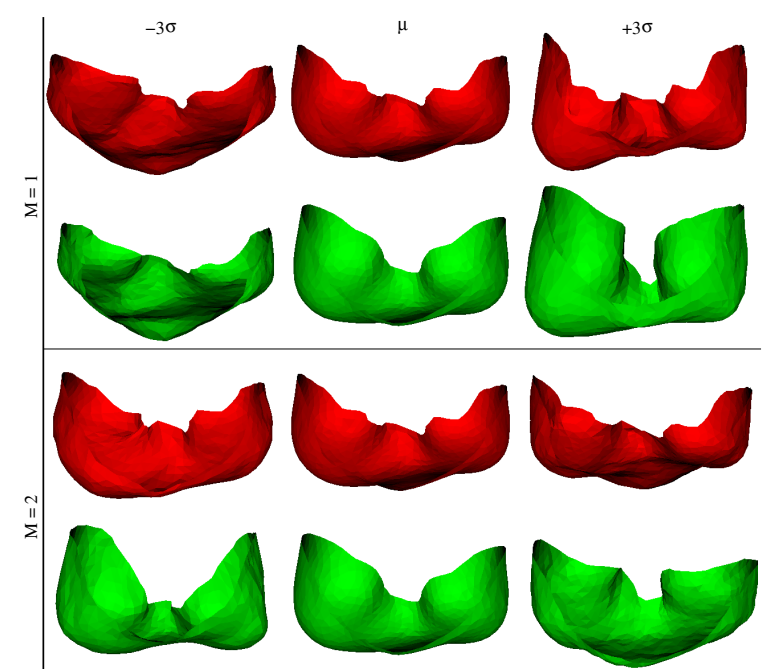

Figure 5. Comparison of the proposed method with SLIDE: The shape space of the PDM created using SLIDE is shown in red (top row for $M=1,2$ ) and the proposed method is shown in green. $M=1,2$ are the first two principal directions, $\mu$ is the mean shape and $\sigma$ is the standard deviation along a principal direction. Note that SLIDE produces landmarks without connecting them into a mesh. Since the landmarks identified are the same for both methods, we apply the same triangulation order to the SLIDE shapes.

In Fig. 5 we show a few significant instances from the deformable shape space described by the PDMs constructed using the proposed method and SLIDE. Particularly, we show the shape instances after deforming the mean shapes along their first two principal directions, respectively. The magnitudes of deformation along these two directions are chosen from $[-3 \sigma,+3 \sigma]$, with $\sigma$ being the standard deviation of the PDM along a principal direction. We include two movies (slide.mpg) and (method.mpg) in the supplemental material to dynamically demonstrate these shape spaces along the first five principal directions. As shown in Fig. 5, it is clear that the two PDMs describe similar shape spaces, but differ in two aspects. First, the shape space described by the PDM of SLIDE allows shape instances with self-intersecting surfaces and unnatural folding in some regions, as is the case for $-3 \sigma$ for $M=2$, while the proposed method always leads to a simple triangle mesh with no selfintersections. Second, it can be observed that the boundary of the diaphragm shape is not well described by the PDM constructed using SLIDE and shows some folding, while the proposed method leads to a smooth boundary. The case of $+3 \sigma$ for $M=1$ in Fig. 5 is an example of such inaccuracy in describing the boundary of the shape. The major reason for this inaccuracy, in SLIDE, is that no care is taken to represent the boundary sufficiently. In fact, in SLIDE there are no constraints that require the landmarks on the boundary of the template shape to only correspond to points on the boundary of the target shape.

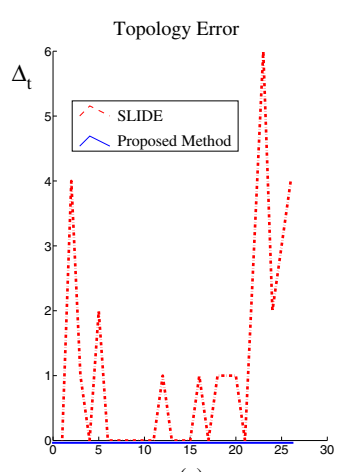

(a)

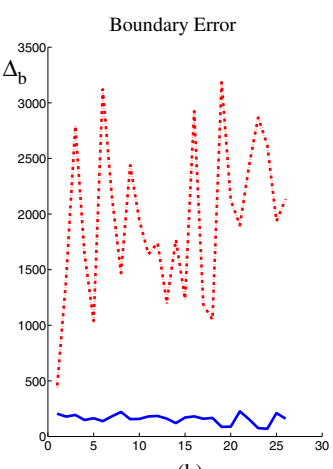

(b)
Figure 6. Comparison of the proposed method with SLIDE: Measures of topology error $\Delta_{t}$ and boundary error $\Delta_{b} . \Delta_{t}=0$ for the proposed method which is described by the horizontal axis in (a). The horizontal axis represents the 26 shape instances.

In this paper we introduce two new measures to check whether the identified landmarks can well represent the topology and the boundary of each diaphragm shape instance. Without correct topology and good representation of the boundary, the identified landmarks cannot well represent the shape instance. First, we define the topology error as:

$$
\begin{aligned}
& \Delta_{t}=\quad \text { Number of triangles in } V_{T} \text { that } \\
& \text { are not topologically consistent with } U_{T} .
\end{aligned}
$$

This measure evaluates the ability of a method to correctly represent the topological homeomorphism of the underlying 
shape. Second, we define the boundary error, as

$$
\Delta_{b}=\sum_{\mathbf{v}_{i} \in V_{B L}}\left\|\mathbf{v}_{i}-\mathbf{v}^{(i)}\right\|
$$

where, $\mathbf{v}^{(i)}$ is the closest point along $V_{B}$ to $\mathbf{v}_{i}$. This measure evaluates the ability of a shape correspondence method to represent the boundary of an open-surface shape. We apply these two new measures to all 26 shape instances and show the measures of $\Delta_{t}$ and $\Delta_{b}$ in Fig. 6. It is clear that the proposed method performs better than SLIDE. Specifically, we can see in Fig. 6 (a) that 13 shape instances have topology inconsistency using SLIDE while the proposed method does not produce any topology errors. Further, in Fig. 6 (b) we can see that the proposed method shows a large improvement over SLIDE for $\Delta_{b}$.
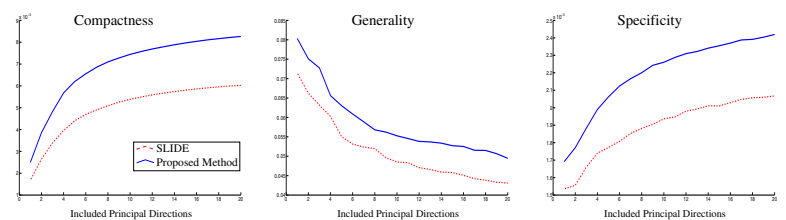

Figure 7. Compactness, generality and specificity measures for the proposed method and SLIDE.

In [29], three quantitative measures are introduced for evaluating the shape correspondence results by examining the compactness, generality and specificity of the constructed PDM. Figure 7 shows these three measures, where we find that SLIDE results in better compactness, generality, and specificity (smaller values) than the proposed method does. This is in contrast to what we obtained above. The major reason is that, without a reference to any ground truth, these three measures only evaluate the properties of the resulting PDM and do not check whether the resulting PDM can really represent the underlying shape. This limitation has been pointed out in several previous works [20,25]. In other words, these three measures are only meaningful when the identified landmarks can well represent the original shape instances, both topologically and geometrically. More specifically, we can clearly see from Fig. 8 that landmarks identified by SLIDE do not correctly model the whole diaphragm by missing the area near the boundary, leading to a PDM with lesser variation and hence a better compactness measure. This also makes the size of the mean shape resulting from SLIDE smaller than that from the proposed method along the vertical direction, which gives SLIDE an undesirable advantage in terms of these three measures. As mentioned above, in this case, the correspondence results from SLIDE are in fact incorrect and the results from these three measures are misleading.

Finally, we asked a medical expert majoring in human anatomy with over 20 years of experience to perform a qualitative evaluation of the proposed method. Specifically, we
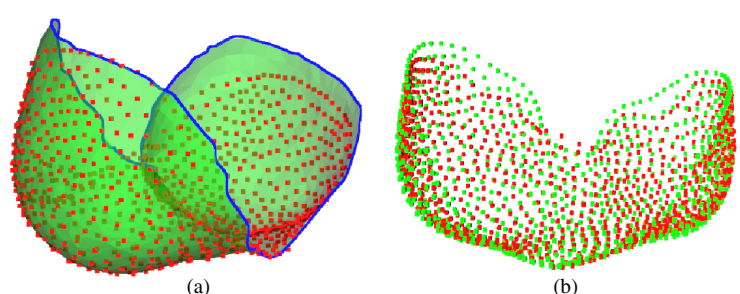

Figure 8. (a) The PDM resulting from SLIDE is unable to model the shape boundary correctly. The identified landmarks (red) are far from the boundary (blue) and hence less variation is observed in the SLIDE PDM. (b) The mean shapes resulting from SLIDE (red) and the proposed method (green) have the same size in the horizontal and depth directions but not the vertical direction giving SLIDE an advantage in the values of compactness, generality and specificity.

asked the expert to comment on the deformable shape space described by the PDM created using the proposed method on the 26 diaphragm shape instances. We changed the magnitudes of deformation along the first five principal directions from $-3 \sigma$ to $+3 \sigma$ to deform the mean shape. The expert identified three major criteria for evaluating this PDM: (a) the smoothness of the diaphragm surface, (b) the symmetry of the surface boundary and, (c) the similarity of the shape space described by the PDM to the general shape of the diaphragm. The expert observed that each shape described by the PDM had a smooth surface reflecting the real case of a diaphragm and expressed satisfaction with this aspect of the PDM. Further, the expert commented that each surface boundary appeared to have good symmetry in the region along the ribs. However, some symmetry was lacking in the part of the boundary near the spine. The expert also asserted that each shape in the deformable shape space can be considered as a naturally occurring case of the diaphragm. Finally, he observed that the mean shape of the PDM can be considered as a common instance of the diaphragm shape. These comments show that the PDM successfully reflects the variation observed in the natural diaphragm shape.

\section{Conclusion}

In this paper, we presented a novel method to address the important problem of open-surface shape correspondence. This method can identify a good triangle mesh from each shape surface such that (a) the vertices of these triangles are the desirable corresponded landmarks and, (b) all meshes are generated using the same connection information. Particularly, we used least squares conformal mapping to facilitate the checking and preservation of the landmark topology consistency, which is critical for constructing a simple, valid mesh without self intersections. In addition, we used the $3 \mathrm{D}$ thin-plate bending energy to model the landmark 
correspondence error and developed an iterative landmark refinement algorithm to reduce this error. We tested the proposed method on 26 human diaphragm shape instances and showed that (a) its performance is better than that of a prior related method and, (b) the PDM constructed using the proposed method leads to a valid representation of the natural variation observed in the diaphragm.

\section{References}

[1] L. Ahfors. Complex Analysis. McGraw-Hill Book Company, New York, 1996.

[2] S. Belongie, J. Malik, and J. Puzicha. Shape matching and object recognition using shape contexts. PAMI, 24(24):509522, April 2002.

[3] F. Bookstein. Principal warps: Thin-plate splines and the decomposition of deformations. PAMI, 11(6):567-585, June 1989.

[4] F. Bookstein. Landmark methods for forms without landmarks: Morphometrics of group differences in outline shape. Medical Image Analysis, 1(3):225-243, 1997.

[5] C. Brechbuhler, G. Gerig, and O. Kubler. Parametrization of closed surfaces for 3-d shape description. CVGIP, 61:154170, 1995.

[6] A. Brett and C. Taylor. A framework for automated landmark generation for automated $3 \mathrm{~d}$ statistical model construction. In IPMI, pages 376-381, 1999.

[7] H. Chui and A. Rangarajan. A new algorithm for non-rigid point matching. In $C V P R$, pages 44-51, 2000.

[8] T. Cootes, A. Hill, C. Taylor, and J. Haslam. The use of active shape models for locating structures in medical images. IVC, 12:355-366, 1994.

[9] T. Cootes, C. Taylor, D. Cooper, and J. Graham. Active shape models - their training and application. CVIU, 61(1):38-59, Jan. 1995.

[10] P. Dalal, B. Munsell, S. Wang, J. Tang, K. Oliver, H. Ninomiya, X. Zhou, and H. Fujita. A fast 3d correspondence method for statistical shape modeling. CVPR, 2007.

[11] R. Davies, C. Twining, T. Cootes, J. Waterton, and C. Taylor. A minimum description length approach to statistical shape modeling. IEEE Trans. Medical Imaging, 21(5):525-537, May 2002.

[12] J. Duchon. Splines minimizing rotation-invariant seminorms in Sobolev space. In Constructive Theory of Functions of Several Variables, Lecture Notes in Mathematics, 571, pages 85-100, 1977.

[13] D. C. V. Essen. Surface-based approaches to spatial localization and registration in primate cerebral cortex. NeuroImage, 23:S97-S107, 2004.

[14] O. Faugeras et al. Variational, geometric, and statistical methods for modeling brain anatomy and function. NeuroImage, 23:Pages S46-S55, 2004.

[15] B. Geiger. Three-dimensional modelling of human organs and its application to diagnosis and surgical planning. In Technical Report: INRIA France, 1993.
[16] G. Gerig, M. Styner, D. Jones, D. Weinberger, and J. Lieberman. Shape analysis of brain ventricles using spharm. In MMBIA, pages 171-178, 2001.

[17] A. Gersho and R. Gray. Vector Quantization and Signal Compression. Kluwer, Boston, 1992.

[18] T. Heimann, I. Wolf, T. Williams, and H.-P. Meinzer. 3d active shape models using gradient descent optimization of description length. In IPMI, 2005.

[19] A. Hill and C. Taylor. A framework for automatic landmark identification using a new method of nonrigid correspondence. PAMI, 22(3):241-251, March 2000.

[20] J. Karlsson and A. Ericsson. A ground truth correspondence measure for benchmarking. ICPR, 3:Pages 568-573, 2006.

[21] A. Kelemen, G. Szekely, and G. Gerig. Elastic model-based segmentation of 3-d neuroradiological data sets. IEEE Trans. Medical Imaging, 18(10):828-839, 1999.

[22] A. Kotcheff and C. Taylor. Automatic construction of eigenshape models by genetic algorithm. In IPMI, pages 1-14, 1997.

[23] M. Leventon, E. Grimson, and O. Faugeras. Statistical shape influence in geodesic active contours. In CVPR, pages 316323, 2000.

[24] B. Levy, S. Petitjean, N. Ray, and J. Maillot. Least squares conformal maps for automatic texture atlas generation. $S I G$ GRAPH, 2002.

[25] B. Munsell, P. Dalal, and S. Wang. Evaluating shape correspondence for statistical shape analysis: A benchmark study. PAMI, 30:Pages 2023-2039, 2008.

[26] A. Pitiot, H. Delingette, A. Toga, and P. Thompson. Learning object correspondences with the observed transport shape measures. In IPMI, pages 25-37, 2003.

[27] T. Richardson and S. Wang. Open-curve shape correspondence without endpoint correspondence. In MICCAI, pages I:17-24, 2006.

[28] C. Shelton. Morphable surface models. IJCV, 38(1):75-91, 2000.

[29] M. Styner, K. Rajamani, L.-P. Nolte, G. Zsemlye, G. Szekely, C. Taylor, and R. Davies. Evaluation of 3D correspondence methods for model building. In IPMI, 2003.

[30] D. Tosun, M. Rettmann, X. Han, X. Tao, C. Xu, S. Resnick, D. Pham, and J. Prince. Cortical surface segmentation and mapping. NeuroImage, 23:S108-S118, 2004.

[31] G. Wahba. Spline Models for Observational Data. Society Industrial and Applied Mathematics, 1990.

[32] S. Wang, X. Ji, and Z.-P. Liang. Landmark-based shape deformation with topology-preserving constraints. In ICCV, pages 923-930, 2003.

[33] S. Wang, T. Kubota, and T. Richardson. Shape correspondence through landmark sliding. In CVPR, pages I-143-150, 2004.

[34] Y. Wang, B. Peterson, and L. Staib. Shape-based 3D surface correspondence using geodesics and local geometry. In CVPR, pages (II)644-651, 2000.

[35] X. Zhou, H. Ninomiya, T. Hara, H. Fujita, R. Yokoyama, H. Chen, M. Kanematsu, and H. Hoshi. Automated identification of diaphragm in non-contrast torso ct images and its application to computer-aided diagnosis systems. Int. J. of Comput. Assist. Radiology and Surgery, 1:366-367, 2006. 